\title{
The impact of COVID-19 on self-management behaviours and healthcare access for people with inflammatory arthritis
}

\author{
Emma Caton ${ }^{1 *} \mathbb{0}$, Hema Chaplin ${ }^{1}$, Lewis Carpenter ${ }^{1}$, Melissa Sweeney ${ }^{1}$, Hsiu Yen Tung ${ }^{1}$, Savia de Souza ${ }^{2}$, \\ James Galloway², Elena Nikiphorou ${ }^{2}$ and Sam Norton ${ }^{1,2}$
}

\begin{abstract}
Background: Inflammatory arthritis (IA) patients have been identified as at greater risk of severe illness from COVID19. It is likely that lockdown restrictions (enforced by the UK government in response to the COVID-19 pandemic) and subsequent changes made to healthcare provision could impact patients'abilities to effectively manage their condition. The aim of this study was to qualitatively explore the impact of COVID-19 on self-management behaviours and healthcare access for people with IA.
\end{abstract}

Methods: Semi-structured interviews were conducted with 21 IA patients in June-July 2020, with nine follow-up interviews in November 2020. Interview schedules were developed with a Patient Research Partner and explored participants' experiences of the COVID-19 pandemic. Interviews were conducted via telephone and analysed using inductive thematic analysis.

Results: Participants were aged between 24 and 79 years (mean $=50.1, \mathrm{SD}=15.8$ ), largely female $(71 \%)$ and White British (86\%). Four initial themes were identified: (1) Impact of COVID-19 on medication adherence, (2) Impact of COVID-19 on physical activity, (3) Impact of COVID-19 on diet, and (4) Impact of COVID-19 on healthcare access and delivery. Subthemes focused on positive and negative changes made to these areas, as well as behaviours which remained consistent. Follow-up interviews highlighted differences in participants' experiences during the two lockdown periods.

Conclusion: COVID-19 has affected patients'abilities to manage their IA. Healthcare professionals need to recognise the ongoing impact of COVID-19 on patient self-management and healthcare access to ensure that adequate understanding and support is available to patients who may have inadequate disease control as a result.

Keywords: COVID-19, Inflammatory arthritis, Qualitative, Self-management, Healthcare access

*Correspondence: emma.caton@kcl.ac.uk

${ }^{1}$ Health Psychology Section, 5th Floor, Bermondsey Wing, Guy's Hospital, Institute of Psychiatry, Psychology and Neuroscience, King's College London, London Bridge, London SE1 9RT, UK

Full list of author information is available at the end of the article

\section{Key messages}

- The first COVID-19 lockdown had positive and negative impacts on physical activity, diet and medication-taking.

- The most common healthcare disruption was the introduction of telephone appointments to replace face-to-face consultations. 
- Rheumatologists need to ensure they support patients who have inadequate disease control following the pandemic.

\section{Background}

The UK government announced the first nationwide lockdown on 23rd March 2020; imposing strict restrictions on movement to prevent the spread of COVID-19 [1]. Inflammatory arthritis (IA) patients were identified as at greater risk of severe illness from COVID-19 and advised to follow strict 'shielding' guidelines. These guidelines encouraged patients to refrain from leaving their home, except for medical appointments, and to get food and medication delivered [2].

The unprecedented nature of the COVID-19 pandemic provided a unique opportunity to explore how people living with IA (e.g. rheumatoid arthritis, psoriatic arthritis, spondyloarthritis, connective tissue disease-related arthritis) are able to manage their condition whilst also observing lockdown restrictions. In order to reduce disease activity, IA patients are often prescribed immunosuppressant disease-modifying anti-rheumatic drugs (DMARDs) [3], which may lead to medication nonadherence in people with rheumatic disease [4] due to attributing higher risk of contracting COVID-19. Furthermore, the closure of all fitness facilities [5] and difficulties accessing food during the pandemic [6], have significantly altered the opportunities available to IA patients to engage in physical activity and healthy eating behaviours. The pandemic has also seen significant changes made to healthcare access and delivery as many face-to-face consultations were cancelled in favour of remote consultations [7].

It is likely that lockdown restrictions and subsequent changes made to healthcare provision, could impact the ability of IA patients to effectively manage their condition. The aim of this study was to qualitatively explore the impact of COVID-19 over the first two UK lockdown periods on self-management behaviours and healthcare access for people with IA.

\section{Methods}

\section{Design and recruitment}

This qualitative study was a sub-study nested within a larger longitudinal survey study (IA-COVID) exploring the long-term impact of COVID-19 on health-related quality of life for people with IA [8].

Participants were recruited via social media and relevant charity organisations. To be eligible for the study, participants needed to be $18+$ years old, living in the UK and diagnosed with an IA condition. Participants were recruited to the qualitative sub-study following completion of the main survey, through additional research consent for contact.

Of the 338 participants from the main survey, 332 provided consent for further contact. People were approached in blocks of 10 until at least 20 had agreed to participate, including representation (i.e. at least 3 people) from each sex, ethnic minority groups, and the following IA condition groups: rheumatoid arthritis, psoriatic arthritis, spondyloarthritis, connective tissue disease-related arthritis. The study included patients who were taking conventional synthetic (cs) and/or biological (b) DMARDs, as well as those who had not been prescribed DMARDs for their condition. Sample size was determined a priori using a pragmatic approach; a sample of approximately 20 participants was deemed both feasible and appropriate given the scope of the study and the resources available to the research team for a medium thematic analysis project conducted as part of a Masterslevel research programme [9].

Informed written consent was obtained from each participant at the beginning of both the survey and telephone interviews.

\section{Materials and procedure}

Semi-structured interviews were conducted between 19th June and 22nd July 2020, with follow-up interviews conducted in November 2020. Interview schedules for both the baseline (see Additional File 1: Table S1) and follow-up interviews (see Additional File 2: Table S2) were developed by the researchers (EC, HC and SN) in collaboration with a Patient Research Partner (SdS).

The interview schedules aimed to explore participants' early experiences of the COVID-19 pandemic. Questions were focused on participants' mental and physical health during the lockdown period, clarity of the messages and guidance received, and impact of COVID-19 on their self-management behaviours and healthcare access.

The interview schedules were piloted with the Patient Research Partner (SdS) and modified appropriately. Patient interviews were conducted in English, via telephone, and lasted 15-30 min. The interviews were audiorecorded, with permission, and transcribed verbatim.

\section{Analysis}

Data was analysed using inductive thematic analysis [10], which allowed for a deep, data-driven exploration into patients' experiences. Transcripts were read and re-read before being systematically coded by the lead researcher (EC). Five transcripts were independently coded by another researcher (HC). Similar codes were collated and organised to form themes and subthemes. These were discussed within the research team to ensure that they were well-developed and representative of the data. 


\section{Results}

\section{Participants}

Twenty-one participants took part in the baseline interviews in June and July 2020, (see Table 1 ). These participants were also invited to complete follow-up interviews in November 2020, nine of which agreed to take part. Participants were aged between 24 and 79 years old (mean $=50.1, \mathrm{SD}=15.8$ ), were mostly female $(71 \%)$ and White British (86\%). Four IA conditions were reflected in the sample (Rheumatoid arthritis: $n=3$; Psoriatic arthritis: $n=8$; Spondyloarthritis: $n=7$; Connective tissue disease-related arthritis: $n=3$ ). Disease duration ranged from $<1$ to 39 years (median $=7, \mathrm{IQR}=11.5$ ). The majority of participants $(n=19)$ had been prescribed csDMARDs (21\%), bDMARDs (31.6\%), or a combination of both $(47.4 \%)$ to help manage their IA.

\section{Themes}

Four main themes were generated from the data: (1) Impact of COVID-19 on medication adherence, (2) Impact of COVID-19 on physical activity, (3) Impact of COVID-19 on diet and (4) Impact of COVID-19 on healthcare access and delivery. A full summary of themes and subthemes can be found in Table 2 .
Theme 1: Impact of COVID-19 on medication adherence During the first two COVID-19 lockdowns, most participants remained adherent to their prescribed medication regimens. However some participants discontinued their medication, with clearer information and reassurance required. Reassuringly, during the second lockdown period, fewer participants reported medication non-adherence.

\section{Adherence to prescribed medication regimen}

The majority of participants did not have any concerns about taking their medication during the pandemic and, as such, continued to take their prescribed medication as usual:

[Has anything changed in regard to taking your medication?] "No... I take my medication every three weeks." (Participant 16, baseline)

Similarly, participants who experienced COVID-19 symptoms, or had been in close contact with someone who had, recognised that it was usually important to stop taking their immunosuppressants for a period of time. As a result, some participants paused their medication regimen in line with usual medication practices and advice:

I'd been in contact with someone with coronavi-

Table 1 Characteristics of interview participants

\begin{tabular}{|c|c|c|c|c|c|}
\hline Participant ID & Sex & Age & IA condition & Ethnicity & Geographical location \\
\hline Participant $1^{*}$ & Female & 70 & Rheumatoid arthritis & White British & West Midlands \\
\hline Participant 2 & Female & 55 & Rheumatoid arthritis & White British & Yorkshire and the Humber \\
\hline Participant 3 & Female & 38 & Rheumatoid arthritis & White British & London \\
\hline Participant 4 & Female & 59 & Psoriatic arthritis & White British & Yorkshire and the Humber \\
\hline Participant 5 & Female & 48 & Psoriatic arthritis & White (any other White background) & Glasgow \\
\hline Participant 6 & Female & 39 & Psoriatic arthritis & White British & South East Wales \\
\hline Participant $7 *$ & Female & 35 & Psoriatic arthritis & White British & South East England \\
\hline Participant $8^{*}$ & Male & 79 & Psoriatic arthritis & White British & London \\
\hline Participant $9^{*}$ & Male & 72 & Psoriatic arthritis & White British & London \\
\hline Participant $10^{*}$ & Male & 61 & Psoriatic arthritis & White British & Yorkshire and the Humber \\
\hline Participant $11^{*}$ & Male & 46 & Psoriatic arthritis & White British & South East England \\
\hline Participant 12 & Female & 67 & Spondyloarthritis & White British & London \\
\hline Participant 13 & Female & 59 & Spondyloarthritis & White British & South East England \\
\hline Participant 14 & Female & 43 & Spondyloarthritis & White British & South East England \\
\hline Participant $15^{*}$ & Female & 31 & Spondyloarthritis & White British & London \\
\hline Participant 16 & Female & 30 & Spondyloarthrits & Mixed (any other Mixed background) & London \\
\hline Participant $17^{*}$ & Female & 24 & Spondyloarthritis & White British & East of England \\
\hline Participant $18^{*}$ & Male & 31 & Spondyloarthritis & White British & South West England \\
\hline Participant 19 & Female & 68 & Connective tissue disease- related arthritis & Black or Black British & West Midlands \\
\hline Participant 20 & Female & 48 & Connective tissue disease-related arthritis & White British & South East England \\
\hline Participant 21 & Male & 49 & Connective tissue disease-related arthritis & White British & West Midlands \\
\hline
\end{tabular}

*Participants who took part in the follow-up interviews in November 2020 
Table 2 Summary of themes and subthemes

\begin{tabular}{|c|c|c|}
\hline Themes & Subthemes & Description \\
\hline \multirow[t]{2}{*}{ Impact of COVID-19 on medication adherence } & Adherence to prescribed medication regimen & $\begin{array}{l}\text { Most participants remained adherent to their } \\
\text { prescribed medication regimens throughout the } \\
\text { pandemic }\end{array}$ \\
\hline & Lack of adequate information and reassurance & $\begin{array}{l}\text { Some participants received very little advice } \\
\text { about taking their medication during the pan- } \\
\text { demic and thus discontinued their medication } \\
\text { regimen }\end{array}$ \\
\hline \multirow[t]{3}{*}{ Impact of COVID-19 on physical activity } & $\begin{array}{l}\text { Increased opportunity to engage in physical } \\
\text { activity }\end{array}$ & $\begin{array}{l}\text { For some participants, the COVID-19 lockdown } \\
\text { provided the time and opportunity to engage in } \\
\text { more physical activity and exercise }\end{array}$ \\
\hline & Shielding guidelines restricting physical activity & $\begin{array}{l}\text { Most participants expressed that the introduction } \\
\text { of 'shielding' guidelines led to a decrease in their } \\
\text { physical activity levels }\end{array}$ \\
\hline & Seasonal changes restricting physical activity & $\begin{array}{l}\text { Many participants expressed that they were una- } \\
\text { ble to engage in outdoor physical activity during } \\
\text { the second lockdown due to the poor weather }\end{array}$ \\
\hline \multirow[t]{3}{*}{ Impact of COVID-19 on diet } & Increased capacity to plan healthy meals & $\begin{array}{l}\text { Some participants expressed that their diet had } \\
\text { improved during the two lockdown periods due } \\
\text { to having the increased time and capacity to plan } \\
\text { and prepare healthy meals }\end{array}$ \\
\hline & $\begin{array}{l}\text { Continuation of pre-pandemic dietary behav- } \\
\text { iours }\end{array}$ & $\begin{array}{l}\text { Some participants did not make any changes to } \\
\text { their diet during the COVID-19 pandemic }\end{array}$ \\
\hline & Diet as emotional regulation & $\begin{array}{l}\text { Most participants stated that their diet had got } \\
\text { worse during the lockdown periods due to an } \\
\text { increase in emotional eating }\end{array}$ \\
\hline \multirow[t]{3}{*}{$\begin{array}{l}\text { Impact of COVID-19 on healthcare access and } \\
\text { delivery }\end{array}$} & Changes to healthcare access and delivery & $\begin{array}{l}\text { All participants experienced changes to } \\
\text { healthcare access during the initial stages of the } \\
\text { COVID-19 pandemic, including the introduction } \\
\text { of telephone appointments to replace face-to- } \\
\text { face consultations }\end{array}$ \\
\hline & $\begin{array}{l}\text { Positive impact of changes to healthcare access } \\
\text { and delivery }\end{array}$ & $\begin{array}{l}\text { Many participants found the introduction of } \\
\text { telephone appointments beneficial }\end{array}$ \\
\hline & $\begin{array}{l}\text { Negative impact of changes to healthcare } \\
\text { access and delivery }\end{array}$ & $\begin{array}{l}\text { Some participants stated that changes to health- } \\
\text { care access had had a negative impact on their } \\
\text { health and wellbeing, especially if their disease } \\
\text { was not well-controlled }\end{array}$ \\
\hline
\end{tabular}

rus and my nurse advised that I just isolate for two weeks anyway and not to take my injection for two weeks from that day. (Participant 17, baseline)

\section{Lack of adequate information and reassurance}

Some participants reported having stopped taking their medication, despite not having symptoms of, or contact with, COVID-19, and without consulting their healthcare team:

\section{...probably slightly hysterical at the start like eve- ryone else or some anxiety y'know about whether I should take it or not...just in case it made me more susceptible to um catch the virus (Participant 11, baseline)}

This quote suggests that one of the main reasons for medication non-adherence was concerns from patients that their medication would make them more susceptible to contacting COVID-19. The term "slightly hysterical" provides an insight into the negative emotions experienced by patients upon having to decide whether to continue with their medication regimen.

Patients who did not have any concerns about taking their medication during the COVID-19 pandemic, noted that this was primarily due to the advice that they had received from their rheumatology team:

I didn't really [have any concerns about taking medication] 'cause my rheumatologist was very clear, um, when she saw me at my first consultation... (Participant 7, baseline)

This suggests that adequate information and reassurance from rheumatology services is important to reduce patients' concerns about taking immunosuppressive medication during the pandemic. Even so, some 
participants felt that they did not receive adequate information regarding their medication and noted that they would have found it beneficial if more advice had been made available to them at the beginning of the pandemic:

I wish there had been advice at the start of the lockdown ... I didn't really have any um advice concerning um my immunosuppressant drugs, you know, whether I should- what the best thing to do was. (Participant 11, follow-up)

\section{Theme 2: Impact of COVID-19 on physical activity}

Physical activity (PA) was another behaviour that was influenced by the pandemic. The initial lockdown provided an opportunity for some IA patients to engage more in PA, whilst for others the restrictions led to a decrease in their exercise levels. Whilst some participants were able to increase their PA when lockdown restrictions eased in August and continue with these behaviours throughout the second lockdown, some reported a further decrease in PA during the second lockdown period due to seasonal changes in the weather.

\section{Increased opportunity to engage in PA}

For some participants, the COVID-19 lockdown had a positive impact on their PA levels:

...I've been consistently doing what I would call maybe moderate with occasional vigorous exercise five times a week which before [lockdown] it was like nothing... (Participant 6, baseline)

Encouragingly, data from the follow-up interviews suggests that many participants were able to maintain these positive exercise behaviours throughout the second lockdown period:

I haven't stopped the exercise that I took up in the first one [lockdown], so I've tried to get a walk in or run in every working day. (Participant 18, follow-up)

These increases in PA levels were directly attributed by participants to lockdown restrictions; the restrictions had lessened their work commitments therefore they had more time to exercise:

Because I weren't at work, I was actually starting to exercise a little more. I started doing Joe Wicks every single day with my little boy. (Participant 17, baseline)

I am doing the things that I always wanted to do but never had the time in terms of exercise. (Participant 15, baseline)

These quotes also demonstrate other positive outcomes from initial lockdown such as ability to spend more time with family. Additionally, lockdown restrictions gave people the opportunity to prioritise themselves over their workload and engage in activities that they have always wanted to do, which may also benefit their mental wellbeing in addition to their physical health.

\section{Shielding guidelines restricting $P A$}

Although some reported increased PA, the majority of participants noted that their PA levels decreased during the lockdown period:

I'm probably doing less physical activity at the moment overall. (Participant 21, baseline)

Participants suggested that the reduction in their PA resulted primarily from the government shielding guidelines:

...if we weren't... in the middle of a pandemic and I wasn't shielding, I'd definitely be going out for a walk a lot more. (Participant 7, baseline)

This shows that, for individuals who usually exercise by walking or participating in other PA outside, the government restrictions greatly impaired their ability to engage in these behaviours and caused their PA levels to reduce.

\section{Seasonal changes restricting $P A$}

Environmental factors were also identified as a barrier to PA during the COVID-19 pandemic. For example, during the second lockdown period, some participants who had previously increased their PA noted that they were now unable to engage in outdoor exercise due to the poor weather:

this second lockdown the weather has been awful generally, so doing exercise has been a little bit more of an effort...I'm not doing nearly as much exercise in this second lockdown as I did in the first. (Participant 10, follow-up)

\section{Theme 3: Impact of COVID-19 on diet}

In relation to eating behaviours, some participants noted that their diet had improved as a result of having an increased capability to plan and prepare healthy food. Some participants were able to maintain their prepandemic dietary behaviours whilst other noted that their diet had got worse due to the emotional challenges of lockdown. For the majority of participants, dietary behaviours remained consistent across the two lockdown periods. 


\section{Increased capacity to plan healthy meals}

Some participants expressed that their diet had improved during the first two lockdown periods due to having more time to plan and prepare healthy meals.

I'm, um, shopping better. I'm planning meals, um, which is making me eat healthier. (Participant 14, baseline)

I have more time to think about healthy options... more time to think about what I'm eating and how I'm cooking. (Participant 15, follow-up)

Changes to food access due to difficulties going to the supermarket or accessing delivery slots also led to improvements in diet, as participants were encouraged to shop more conscientiously:

...because I wasn't going to the shops... I found a fruit and veg supplier... I got these amazing fruit and veg boxes. (Participant 15, baseline)

\section{Continuation of pre-pandemic dietary behaviours}

Several participants did not experience any changes to their diet during the pandemic.

We eat reasonably healthy...I think our diet's pretty much the same. (Participant 2, baseline)

This suggests that those who were already engaging in healthy eating behaviours pre-pandemic were able to maintain this throughout the lockdown periods.

\section{Diet as emotional regulation}

Most participants stated that their diet had been worse during the first lockdown period, especially in relation to the type and amount of food that they were consuming:

We were eating probably food that we wouldn't normally eat; we were a little less strict... we were comfort eating. (Participant 13, baseline)

The term 'comfort eating' implies that participants may be using food to regulate their emotional responses to COVID-19. Indeed, as the pandemic continued into a second lockdown, 'comfort eating' behaviours also continued, causing some participants to gain weight:

I mean I've been putting weight on, I have put about a stone on across lockdown, and I mean that's entirely due really to comfort eating and boredom. (Participant 1, follow-up)

\section{Theme 4: Impact of COVID-19 on healthcare access and delivery}

The COVID-19 pandemic saw several changes being made to how healthcare services, such as rheumatology appointments and blood tests, were delivered. These changes were perceived both positively and negatively.

\section{Changes to healthcare access and delivery}

The most commonly reported change was the introduction of telephone appointments to replace face-to-face consultations. Remote approaches were applied to most healthcare services, including both rheumatology and GP consultations:

...various hospital appointments, um, with the consultants were over the phone rather than me going to the hospital... (Participant 8, baseline)

Secondary care (e.g. ophthalmology, podiatry) and blood test services were also disrupted during the pandemic, with many appointments being cancelled or postponed until after the lockdown period:

I can't reach to cut my own toenails, um, so that obviously going to, you know, the foot clinic in the [local hospital] has all been cancelled. (Participant 12, baseline)

Positive impact of changes to healthcare access and delivery Some participants found the healthcare changes to be very beneficial. For example, Participant 6 noted that during their telephone consultations, they perceived healthcare professionals to be more receptive to their concerns:

... since lockdown they've been a lot more attentive and give me more time and listened a bit more to whatever it is I'm going through. (Participant 6, baseline)

It was also expressed that the use of telephone consultations may have economic benefits longer term for the healthcare system as well as saving time for both the service and patients:

I think it's a very good idea to, um, to do this [telephone appointments]... it must save a lot of time and money and also it saves a lot of, um, time from the patient's perspective. (Participant 10, baseline) There are a lot of issues that you can sort out over the phone... you do get things done quicker now. (Participant 17, follow-up)

\section{Negative impact of changes to healthcare access and delivery} In some circumstances, the changes made to healthcare access had a negative impact on IA patients. For example, some participants reported experiencing increased health-related anxiety as a result of their blood monitoring being cancelled or postponed: 
I've not had a blood test now in months so I don't know how, y'know, my liver and my kidneys, um, are responding. I don't know what my inflammatory levels are like...it would be nice to have confirmation, um, of that. (Participant 1, baseline)

Several participants also found the introduction of telephone appointments to replace face-to-face consultations unhelpful:

I don't think that just speaking to somebody on the phone you can, you know, they can really get to the gist of what's happening. I think they need to see me, um, and I didn't really find the telephone consultations helpful at all. (Participant 5, baseline)

Indeed, the lack of physical examination generated concerns for patients that decisions regarding their health were being made based on information from previous appointments:

...she obviously couldn't see me, she couldn't look at me she couldn't feel my joints. She couldn't, you know um, do anything apart from base her judgement on what she knew. (Participant 4, baseline)

Even though several IA patients found the telephone consultations to be unhelpful, they understood why changes to healthcare access had to be made during the pandemic:

... I completely understood why we had to do that... but just with the situation I was in [active disease] didn't really help that much. (Participant 3, baseline)

The fact that Participant 3 found the telephone consultation unhelpful due to "the situation I was in" implies that effectiveness of telephone appointments may be linked to a patient's disease activity. Indeed, Participant 2 expressed that they would have found the telephone appointments less challenging if their IA were more controlled:

I think it would be alright if I was quite stable [dis- ease control] but because I'm not stable at the moment it's quite frustrating. (Participant 2, baseline)

\section{Discussion}

The purpose of this study was to explore the impact of COVID-19 on self-management and healthcare access for people with IA during the first two UK lockdown periods. Findings suggest that COVID-19 has had both positive and negative impacts on behaviours such as exercise, diet, and medication-taking, many of which remained consistent over-time. The analysis also helped identify some of the changes made to healthcare services during the pandemic and how these changes were received by IA patients. Based on these findings, recommendations to support IA patients during the COVID-19 pandemic have been suggested (see Table 3).

In relation to $\mathrm{PA}$, some participants reported that they were able to engage in more PA during the pandemic whilst others reported their PA levels had decreased. These changes in exercise behaviours were mostly attributed to the lockdown restrictions and shielding guidelines issued by the UK government. Data from our larger IA-COVID quantitative study [8] suggests that approximately half of IA patients reduced their PA levels during the two lockdown periods. Current research supports the notion that lockdown measures have led to a decrease in PA for clinically extremely vulnerable populations [11, 12]. In relation to rheumatic diseases, physical inactivity resulting from the COVID-19 pandemic may lead to worsened disease activity, increased symptoms of mental distress and a poorer overall quality of life [13]. Nevertheless, for some participants, being instructed to stay at home during the pandemic created the opportunity to engage in more PA because they were spending less time preoccupied with other commitments. Rheumatologists may want to emphasise the benefits of prioritising PA, especially home-based exercises, so that IA patients continue engage in these behaviours.

Table 3 Recommendations to support inflammatory arthritis (IA) patients during the COVID-19 pandemic

Recommendations

Continue to emphasise the importance of engaging in positive health behaviours (e.g. physical activity, diet, medication adherence) for the management of IA

Provide time-management advice to patients who want to prioritise engagement in positive health behaviours (e.g. engaging in physical activity, planning/preparing healthy meals)

Provide home-based exercise programmes to encourage patients to engage in physical activity at home

Signpost patients to mental health resources to help support patients who may be experiencing emotional challenges as a result of the pandemic

Encourage open discussions and provide reassurance to patients who may have concerns about taking their medication during the pandemic

Offer routine telephone appointments to patients who feel that their IA condition is well-controlled 
In addition, the UK lockdowns have also provided an opportunity for IA patients to engage in healthy eating behaviours; several participants noted that their diet had improved due to having more time to plan and prepare meals. Nonetheless, for most participants the lockdowns led to a worsening of diet. A cross-sectional survey study found that poor diet during the COVID-19 lockdown can be linked to a more negative mood [14]. In our study, several participants noted that they increased their consumption of unhealthy 'comfort' foods during the pandemic as a way of dealing with difficult emotions such as anxiety or boredom.

Regarding medication, the findings suggest that, for the majority of participants medication adherence was not influenced. Nevertheless, some participants did express discontinuing their medication due to concerns that it would make them more vulnerable to COVID-19. It is possible that these concerns may have been influenced by the high-levels of uncertainty regarding the use of DMARDs in the early stages of the pandemic; healthcare professionals were initially concerned that the use of immunosuppressive or immunomodulatory agents may predispose patients to severe COVID-19 infection [15]. Reassuringly, emerging literature suggests that the use of DMARDs is not associated with an increased risk of severe illness from COVID-19, and therefore do not need to be discontinued unless in the case of suspected or confirmed COVID-19 infection [16, 17]. In this study, adequate reassurance from healthcare professionals led to many participants continuing with their medication regimen during the pandemic. It is therefore necessary to ensure that the importance of medication adherence is more-widely communicated to IA populations to prevent the negative consequences of non-adherence.

Participants reported experiencing severe disruptions to healthcare services during the first lockdown period, most notably, the introduction of telephone appointments to replace face-to-face consultations [18]. Whilst some participants found the telephone appointments beneficial, several participants said that they found these consultations unhelpful because they were unable to be physically assessed. The findings imply that patient satisfaction with telephone appointments may be linked to disease activity, with patients with poor disease control finding the telephone appointments most ineffective. Healthcare services may want to consider offering routine telephone appointments as an option for patients who feel that their IA condition is well-controlled. Patient preference and perceived disease-activity should be taken into consideration when triaging for face-to-face or telephone consultations.

A limitation of this study is the lack of ethnic diversity within the sample. Only $14 \%$ of participants at baseline were from Black, Asian or Minority Ethnic (BAME) groups, which is not reflective of the ethnic diversity present in the UK. Given that BAME communities have been disproportionately affected by COVID-19 [19] and thus are likely to have different COVID-related experiences, findings from this study may not be representative of the wider population.

Furthermore, this study also lacks geographical diversity. In England, parts of the Midlands and Northern England (East Midlands, North West and North East) were not represented in the sample. Data from the Office for National Statistics suggests that COVID-19 infection rates varied across England, with North West England often experiencing higher infection rates, and thus greater lockdown restrictions, compared to other regions [20]. As such, the results from this study may not be reflective of the experiences of patients who reside outside the regions represented in this study.

Another limitation of the study is that only a relatively small number of rheumatoid arthritis (RA) patients were included in the sample, despite RA being the most common form of IA [21]. Due to the different disease experiences associated with each IA condition, the lack of RA patients in the study may impact the representativeness of the findings when considered in relation to the wider IA population. Nevertheless, the relatively large number of psoriatic arthritis (PsA) patients included in the sample is a strength of the study, as currently little research has been conducted exploring the experiences of PsA patients during the pandemic.

Findings from this study suggest that COVID-19 had a substantial impact on patients' abilities to manage their IA condition. Future research would need to consider the longer-term impact of these behaviour changes on the health and wellbeing of IA patients and the impact this may have on public health services. Overall, healthcare professionals need to recognise the impact of COVID19 on patient self-management and healthcare provision to ensure that adequate understanding and support in future is available to patients who may have inadequate disease control as a consequence.

\begin{abstract}
Abbreviations
BAME: Black, Asian and Minority Ethnic; bDMARDs: Biological disease-modifying anti-rheumatic drugs; csDMARDs: Conventional synthetic disease-modifying anti-rheumatic drugs; COVID-19: Coronavirus disease 2019; DMARDs: Disease-modifying anti-rheumatic drugs; IA: Inflammatory arthritis; PA: Physical activity; PsA: Psoriatic arthritis; RA: Rheumatoid arthritis.
\end{abstract}

\section{Supplementary Information}

The online version contains supplementary material available at https://doi. org/10.1186/s41927-021-00231-1.

Additional file 1. Table S1: Baseline interview schedule. 
Additional file 2. Table S2: Follow-up interview schedule.

\section{Acknowledgements}

The authors would like to thank all participants who took part in this study.

\section{Authors' contributions}

SN, LC, HC, MS, HT, JG, EN and SdS conceptualised the study. Interviews were organised, conducted and transcribed by EC. EC, HC and SN analysed and interpreted the data. All authors read and approved the final manuscript.

\section{Funding}

Funding was provided by MQ and Versus Arthritis Fellowship (jointly funded) (Grant No. MQ16IP18).

\section{Availability of data and materials}

The data that support the findings of this study are available from the corresponding author, $\mathrm{EC}$, upon reasonable request.

\section{Declarations}

\section{Ethics approval and consent to participate}

Ethical approval was obtained from King's College London Research Ethics Committee (LRS-19/20-18186). Informed written consent was obtained from all participants prior to the interviews being conducted. Methods were performed and reported in accordance with the Standards for Reporting Qualitative Research (SRQR) guidelines.

\section{Consent for publication}

Not applicable.

\section{Competing interests}

The authors declare no competing interests.

\section{Author details}

${ }^{1}$ Health Psychology Section, 5th Floor, Bermondsey Wing, Guy's Hospital, Institute of Psychiatry, Psychology and Neuroscience, King's College London, London Bridge, London SE1 9RT, UK. ${ }^{2}$ Centre for Rheumatic Diseases, King's College London, London, UK.

Received: 7 June 2021 Accepted: 31 August 2021

Published online: 18 October 2021

\section{References}

1. lacobucci G. COVID-19: UK lockdown is "crucial" to saving lives, say doctors and scientists. BMJ. 2020;368:1204. https://doi.org/10.1136/bmj. m1204

2. Public Health England. Guidance on shielding and protecting people who are clinically extremely vulnerable from COVID-19. https://www.gov uk/government/publications/guidance-on-shielding-and-protectingextremely-vulnerable-persons-from-covid-19. Accessed 26 June 2020

3. National Institute for Health and Care Excellence (NICE). Rheumatic disease, suppressing drugs. https://bnf.nice.org.uk/treatment-summary/ rheumatic-disease-suppressing-drugs.html. Accessed 5 Feb 2021.

4. Venerito V, Lopalco G, lannone F. COVID-19, rheumatic diseases and immunosuppressive drugs: an appeal for medication adherence. Rheumatol Int. 2020;40(5):827-8. https://doi.org/10.1007/s00296-020-04566-9.

5. Cabinet Office \& Ministry of Housing, Communities and Local Government. Closing certain businesses and venues in England. https://www. gov.uk/government/publications/further-businesses-and-premises-toclose\#history. Accessed 1 July 2020.
6. Nicola M, Alsafi Z, Sohrabi C, Kerwan A, Al-Jabir A, losifidis C, et al. The socio-economic implications of the coronavirus pandemic (COVID-19): a review. Int J Surg. 2020;78:185-93. https://doi.org/10.1016/J.IJSU.2020.04. 018.

7. Nune A, lyengar K, Ahmed A, Sapkota H. Challenges in delivering rheumatology care during COVID-19 pandemic. Clin Rheumatol. 2020:39:2817-21. https://doi.org/10.1007/s10067-020-05312-z.

8. Sweeney M, Carpenter L, de Souza S, Chaplin H, Tung H, Caton E, et al. The impact of COVID-19 on clinical care, self-management, and mental health of patients with Inflammatory Arthritis. Manuscript submitted for publication. 2021.

9. Braun V, Clarke V. Successful qualitative research: a practical guide for beginners. London: Sage; 2013.

10. Braun V, Clarke V. Using thematic analysis in psychology. Qual Res Psychol. 2006;3(2):77-101. https://doi.org/10.1191/1478088706qp063oa.

11. McAuley H, Hadley K, Elneima O, Brightling CE, Evans RA, Steiner MC, et al. COPD in the time of COVID-19: an analysis of acute exacerbations and reported behavioural changes in patients with COPD. ERJ Open Research. 2020. https://doi.org/10.1183/23120541.00718-2020.

12. Radtke T, Haile SR, Dressel H, Benden C. Recommended shielding against COVID-19 impacts physical activity levels in adults with cystic fibrosis. J Cystic Fibrosis. 2020. https://doi.org/10.1016/j.jcf.2020.08.013.

13. Pinto AJ, Dunstan DW, Owen N, Bonfá E, Gualano B. Combating physical inactivity during the COVID-19 pandemic. Nat Rev Rheumatol. 2020;16:347-8. https://doi.org/10.1038/s41584-020-0427-z.

14. Ingram J, Maciejewski G, Hand CJ. Changes in diet, sleep and physical activity are associated with differences in negative mood during COVID19 lockdown. Front Psychol. 2020;11: 588604. https://doi.org/10.3389/ fpsyg.2020.588604.

15. Thng ZX, De Smet MD, Lee CS, Gupta V, Smith JR, McCluskey PJ, et al. COVID-19 and immunosuppression: a review of current clinical experiences and implications for ophthalmology patients taking immunosuppressive drugs. Br J Ophthalmol. 2021;105:306-10. https://doi.org/10. 1136/bjophthalmol-2020-316586.

16. Favalli EG, Bugatti S, Klersy C, Biggioggero M, Rossi S, De Lucia O, et al. Impact of corticosteroids and immunosuppressive therapies on symptomatic SARS-CoV-2 infection in a large cohort of patients with chronic inflammatory arthritis. Arthritis Res Ther. 2020;22:290. https://doi.org/10. 1186/s13075-020-02395-6.

17. Roongta R, Ghosh A. Managing rheumatoid arthritis during COVID19. Clin Rheumatol. 2020;39:3237-44. https://doi.org/10.1007/ s10067-020-05358-z.

18. Reuter K, Deodhar A, Makri S, et al. The impact of the COVID-19 pandemic on people with rheumatic and musculoskeletal diseases: insights from patient generated data on social media. Rheumatology. 2021;6:66.

19. Aldridge RW, Lewer D, Katikireddi SV, Mathur R, Pathak N, Burns R. Black, Asian and Minority Ethnic groups in England are at increased risk of death from COVID-19: indirect standardisation of NHS mortality data. Welcome Open Res. 2020. https://doi.org/10.12688/wellcomeopenres. 15922.2 .

20. Office for National Statistics. Coronavirus (COVID-19) Infection Survey, UK Statistical bulletins. https://www.ons.gov.uk/peoplepopulationandc ommunity/healthandsocialcare/conditionsanddiseases/bulletins/coron aviruscovid19infectionsurveypilot/previousReleases?page=4. Accessed 28 July 2021.

21. Scott IC, Galloway JB, Scott DL. Inflammatory arthritis in clinical practice. 2nd ed. London: Springer; 2015.

\section{Publisher's Note}

Springer Nature remains neutral with regard to jurisdictional claims in published maps and institutional affiliations. 